\title{
ON THE RELATIONSHIP BETWEEN HAUSDORFF DIMENSION AND METRIC DIMENSION
}

\author{
A. C. Vosburg
}

The definitions of the Hausdorff dimension $\operatorname{dim}_{h} X$, upper metric dimension $\overline{\operatorname{dim}} X$ and lower metric dimension $\operatorname{dim} X$ of a metric space $X$ all depend upon asymptotic characteristics of diameters of sets in covers of $X$. We relate these notions. First we note that $\operatorname{dim}_{h} X \leq \underline{\operatorname{dim}} X$ holds for all totally bounded metric spaces $X$, while on the other hand there exist perfect subsets $A$ of $[0,1]$ satisfying $\operatorname{dim}_{h} A=0$ and $\operatorname{dim} A=1=\operatorname{dim}$ $[0,1]$. Finally we show that there exist perfect subsets $S$ of $\overline{[0,1]}$ which satisfy $\operatorname{dim}_{h} S=0$ and $\overline{\operatorname{dim}} S=1$ even when strong local conditions are imposed.

The notions of Hausdorff dimension (see 1,2) and metric dimension (see 5 p. 296, 8) are closely related; in fact most compact metric spaces encountered in analysis have the same Hausdorff and metric dimensions. In this paper we investigate some aspects of the relationship between these two concepts.

By the Hausdorff dimension of a subset $E$ of a metric space is meant the number $\operatorname{dim}_{h} E=\sup \left\{p: \mu_{p}^{*}(E)=+\infty\right\}$, where $\mu_{p}^{*}(E)$ is defined to be $+\infty$ if $p=0$ and $\mu_{p}^{*}(E)=\sup _{\varepsilon>0} l(E, p ; \varepsilon)$ if $p>0$,

$$
\begin{gathered}
l(E, p ; \varepsilon)=\inf \left\{\sum_{i=1}^{+\infty}\left(\operatorname{diam} E_{i}\right)^{p}: E \subset \bigcup_{i=1}^{+\infty} E_{i}, \operatorname{diam} E_{i} \leqq \varepsilon\right. \text { for each } \\
i=1,2, \cdots\} .
\end{gathered}
$$

For each totally bounded subset $A$ of a metric space (i.e. each subset which for each $\varepsilon>0$ can be covered by a finite number of sets of diameter not exceeding $\varepsilon$ ) the upper metric dimension $\overline{\operatorname{dim}} A$ and lower metric dimension $\operatorname{dim} A$ of $A$ are defined as follows (all logarithms have base 2):

$$
\overline{\operatorname{dim}} A=\varlimsup_{\varepsilon \rightarrow 0+}\left(\log N_{\varepsilon}(A)\right) / \log \left(\varepsilon^{-1}\right)
$$

and

$$
\underline{\operatorname{dim}} A=\underline{\lim }\left(\log N_{\varepsilon}(A)\right) / \log \left(\varepsilon^{-1}\right),
$$

where, for each $\varepsilon>0, N_{\varepsilon}(A)$ denotes the smallest number of sets in any cover of $A$ by sets of diameter not exceeding $2 \varepsilon$. It is customary (see 5, p. 280) to abbreviate $\log N_{\varepsilon}(A)$ by $H_{\varepsilon}(A)$; this function has 
been called (see 5, p. 280) the $\varepsilon$-entropy (or metric entropy) function of $A$. When $\operatorname{dim} A=\overline{\operatorname{dim}} A$ this common value is called the metric

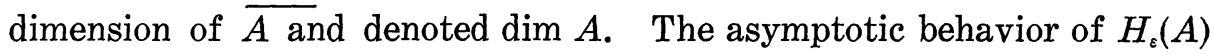
as $\varepsilon \rightarrow 0+$ in some sense reflects the massiveness of $A$, and various applications of this notion have appeared in recent years (see 3, 5, 7, 10). For a tour of the subject of metric entropy see Lorentz $(6,7)$.

We will use the elementary inequality $N_{\varepsilon}(A) \geqq M_{28}(A)$ which is valid (see 6, p. 151) for all totally bounded sets $A$, where $M_{\varepsilon}(A)$ denotes, for each $\varepsilon>0$, the maximal number of points in any subset $S$ of $A$ having the property that each pair of points of $S$ are separated by distance greater than $\varepsilon$.

$\operatorname{dim} A \leqq \overline{\operatorname{dim}} A \leqq \operatorname{dim} A$ being obvious whenever meaningful, we wish to further compare $\operatorname{dim}_{h} A, \operatorname{dim} A, \overline{\operatorname{dim}} A, \operatorname{dim} A$, and investigate some related questions. First (Theorem 1) we will note from elementary considerations that the inequality

$$
\operatorname{dim}_{h} A \leqq \underline{\operatorname{dim} A}
$$

holds for each totally bounded subset $A$ of a metric space. On the other hand we will note that there exist (even perfect) subsets $A$ of $[0,1]$ for which

$$
\operatorname{dim}_{h} A=0 \text { and } \operatorname{dim} A=\operatorname{dim}[0,1]=1 .
$$

Our main result (Theorem 2) involves looking more deeply into the relationship between (4) and (5) by imposing certain additional local conditions.

2. Theorem 1. Let $A$ be a totally bounded subset of a metric space $X$. Then $A$ satisfies (4).

Proof. Let $\operatorname{dim} A=s \geqq 0$, and assume $\delta>0$. By the definition (3), there exists a sequence $\varepsilon_{1}, \varepsilon_{2}, \cdots$ which decreases to zero such that $\log N_{\varepsilon_{n}}(A) \leqq(s+\delta) \log \left(\varepsilon_{n}^{-1}\right)$, and hence $N_{\varepsilon_{n}}(A) \leqq\left(1 / \varepsilon_{n}\right)^{s+\delta}$ for each $n=1,2, \cdots$. This implies the existence of a family of no more than $\left(1 / \varepsilon_{n}\right)^{s+\delta}$ sets of diameter not exceeding $2 \varepsilon_{n}$ which covers $A$. Taking $\varepsilon=2 \varepsilon_{n}$ in the infimum $l$ in (1), this yields

$$
l\left(A, p ; 2 \varepsilon_{n}\right) \leqq\left(1 / \varepsilon_{n}\right)^{s+\delta}\left(2 \varepsilon_{n}\right)^{p} \leqq 2^{p} \text { if } p \geqq s+\delta .
$$

Hence $\mu_{p}^{*}(A) \leqq 2^{p}<+\infty$ if $p \geqq s+\delta$, which, as desired, implies $\operatorname{dim}_{h} A \leqq s$.

While Theorem 1 states that the Hausdorff dimension of a compact set $A$ must be small if the $\varepsilon$-entropy of $A$ approaches $+\infty$ sufficiently slowly, i.e., if $\operatorname{dim} A$ is small, it is natural to ask what can be said 
in the converse direction. Quite elementary considerations show that the reverse inequality need not hold. In fact, it is easy to construct (even perfect) sets $A$ of real numbers for which (5) holds. For example the range $R$ of the sequence $\left\{a_{n}\right\}$ defined by

$$
a_{1}=10, a_{k}=a_{k-1}-1 /\left(k \log ^{2} k\right) \text { if } k>1
$$

satisfies (5), and $R$ can be used to build a perfect set satisfying (5). An example is any perfect set $A$ defined by $A=\bigcup_{k=1}^{+\infty} A_{k} \cup\left\{\lim _{k \rightarrow+\infty} \mathrm{a}_{k}\right\}$, where each set $A_{k}$ is taken to be a perfect set satisfying

$$
\operatorname{dim}_{k} A_{k}=0, \inf A_{k}>a_{k+1}+(1 / 2)\left(a_{k}-a_{k+1}\right) \text { for } k=1,2, \cdots
$$

and

$$
\sup A_{k}<a_{k-1}-(1 / 2)\left(a_{k-1}-a_{k}\right) \text { for } k=2,3, \cdots \text {. }
$$

While in a sense this answers the converse question to Theorem 1 , much is left to be desired, for the largeness of $\operatorname{dim} A$ is really a consequence of that of $\operatorname{dim} R$, and $R$ is only a countable set. We show that the differences between the notions of Hausdorff and metric dimensions run deeper than might be suggested by the above. To do this we introduce the notion of the metric dispersion of a totally bounded subset of a metric space.

Definition 1. Let $A$ be a totally bounded subset of a metric space and let $x_{0} \in A$. Then by the upper metric dimension of $A$ at $x_{0}$, denoted $\overline{\operatorname{dim}}\left(A, x_{0}\right)$, is meant the number

$$
\overline{\operatorname{dim}}\left(A, x_{0}\right)=\inf \left\{\varlimsup_{\varepsilon \rightarrow 0+} H_{\varepsilon}(A \cap U) / \log \left(\varepsilon^{-1}\right): U \varepsilon \boldsymbol{U}\left(x_{0}\right)\right\}, \text { where } \boldsymbol{U}\left(x_{0}\right)
$$

denotes the class of open neighborhoods of $x_{0}$.

Definition 2. By the metric dispersion of $A$, denoted disp $A$, is meant $\left.\operatorname{disp} A=\inf _{x_{0} \& 4} \overline{(\operatorname{dim}}\left(A, x_{0}\right)\right)$.

THEOREM 2. There exist perfect sets $S$ of real numbers satisfying

$$
\operatorname{dim}_{h} S=0 \text { and } \operatorname{disp} S=1 .
$$

Proof. Abbreviating $2^{a}$ by $\exp a$, we let

$$
\begin{aligned}
\delta_{1}=1, \delta_{n} & =\exp \left\{-(n !)^{2}\right\} \\
\varepsilon_{n} & =\exp \left\{-n((n-1) !)^{2}\right\} \\
m_{n} & =\exp \left\{(n-2)((n-1) !)^{2}\right\}, n=2,3, \cdots .
\end{aligned}
$$

For each $n=3,4, \cdots$ and each closed interval $I=[a, b]$ of length at 
A. C. VOSBURG

least $\delta_{n-1}$, we denote by $F(I ; n)$ the subset of $I$ which is the union of all intervals of the family

$$
\Im(I ; n)=\left\{\left[a+i\left(\delta_{n}+\varepsilon_{n}\right), a+i\left(\delta_{n}+\varepsilon_{n}\right)+\delta_{n}\right]: i=0,1, \cdots, m_{n}-1\right\} \text {. }
$$

From (7) it follows that

$$
\delta_{n} \leqq \varepsilon_{n}
$$

and

$$
m_{n}\left(\delta_{n}+\varepsilon_{n}\right) \leqq 2 m_{n} \varepsilon_{n} \leqq \delta_{n-1},
$$

so indeed $F(I ; n)$ is contained in $I$. Further, for each $I$ and $n$, the distance between any two intervals of $\Im(I ; n)$ is no smaller than $\varepsilon_{n}$, and the distance between any pair of left end points of intervals of $\Im(I ; n)$ is no smaller than $\delta_{n}+\varepsilon_{n}>\varepsilon_{n}$.

Now we can define the desired set $S$ as a generalized Cantor set. Let the sequence $\left\{A_{n}\right\}_{n=1}^{+\infty}$ of sets be defined by

$$
A_{1}=[0,1], A_{n}=\cup\left\{F(C ; n): C \in \underset{\sim}{A_{n-1}}\right\} \text { if } n \geqq 2,
$$

where, for each $n=1,2, \cdots,{\underset{\sim}{n}}_{n}$ denotes the family of all component intervals of $A_{n}$ (the components of each $A_{n}$ are intervals of length $\delta_{n}$ ). For $S$ we take $S=\bigcap_{n=1}^{+\infty} A_{n}$.

Since each $A_{n}$ has $\prod_{k=1}^{n} m_{k}$ components, each of length $\delta_{n}$, to prove that $\operatorname{dim}_{h} S=0$ it suffices to observe that from (7) it follows that

$$
\lim _{n \rightarrow+\infty}\left\{\left(\prod_{k=1}^{n} m_{k}\right) \delta_{n}^{p}\right\}=0 \text { for each } p>0 \text {. }
$$

To establish disp $S=1$, we consider any open interval $I$ such that $S \cap I$ is nonempty, and estimate $N_{\varepsilon}(S \cap I)$ from below. If $n$ is sufficiently large, $I$ contains a component interval $[c, d]$ of $A_{n-1}$, and hence also contains all intervals of $\Im([c, d] ; n)$. If we let $\underset{\sim}{C \text { denote a }}$ cover of $F([c, d] ; n)$ by sets of diameter not exceeding $2 \varepsilon, \varepsilon=(1 / 2) \varepsilon_{n}$, then $C$ covers also the set of left end points of the intervals of $\Im([c, \tilde{\mathrm{d}}] ; n)$, and no covering set among the sets of $\underset{\sim}{C}$ covers more than one such endpoint. Hence the number of covering sets of the family $\underset{\sim}{C}$ cannot be less than $m_{n}$. This implies that

$$
N_{\left(\varepsilon_{n} / 2\right)}(S \cap I) \geqq m_{n}
$$

holds for all sufficiently large $n$. Since (7) implies that

$$
\left(\log m_{n}\right) / \log \left(2 / \varepsilon_{n}\right)=(n-2)((n-1) !)^{2} /\left(n((n-1) !)^{2}\right) \rightarrow 1 \text { as } n \rightarrow+\infty,
$$

we have, from (8),

$$
\varlimsup_{\varepsilon \rightarrow 0+} N_{\varepsilon}(S \cap I) / \log \left(\varepsilon^{-1}\right) \geqq 1
$$


Since this limit superior cannot be greater than 1 , we have disp $S=1$, which completes the proof of the theorem.

It remains an open question whether there exist perfect sets $S$ satisfying $\operatorname{dim}_{h} S=0, \operatorname{dim}(S, x)>0$ for all (or at least most, in some sense) of the points $x$ of $S$, where $\operatorname{dim}(S, x)$ is defined analogously to $\overline{\operatorname{dim}}(S, x)$ in Definition 1.

In conclusion we mention that Theorem 2 leaves open the question as to whether for two sets $A, B$ with $\operatorname{dim}_{h} A \leqq \operatorname{dim}_{h} B$ any inequality necessarily follows for $\operatorname{disp} A$, disp $B$. The answer is in the negative; that is, for each $\omega, 0<\omega<1$, there are perfect sets $A, B$ of real numbers such that $\operatorname{dim}_{h} A<\operatorname{dim}_{h} B=\omega$ and $\omega=\operatorname{disp} B<\operatorname{disp} A=1$. This follows from Theorem 2 together with the fact that for each $\omega, 0<\omega<1$, there exist compact perfect sets $B$ such that $\operatorname{dim}_{h} B=\omega$ and $H_{\varepsilon}(B) \sim \omega \log 1 / \varepsilon$. Such sets $B$ are constructed in Hausdorff's paper (see [1], § 10).

The author is very much indebted to his teacher, Professor George Lorentz, for helpful conversations concerning this paper.

\section{REFERENCES}

1. F. Hausdorff, Dimension and outer Measure, Math. Ann. 79 (1919), 157-119.

2. W. Hurewicz and H. Wallman, Dimension Theory, Princeton University Press, Princeton, 1941.

3. A. N. Kolmogorov, Asymptotic characteristics of some completely bounded metric spaces, Dokl. Akad, Nauk SSSR 108 (1956) 585-589.

4. A. N. Kolmogorov and V.M. Tihomirov, e-entropy and $\varepsilon$-capacity of sets in function spaces, Uspehi Mat. Nauk (86), 14 (1959), 3-86.

5. —, E-entropy and e-capacity of sets in functional spaces, Amer. Math. Soc. Translations (2) 17, 277-364 (English translation of 4).

6. G. G. Lorentz, Approximation of functions, Holt, Rinehart and Winston, New York, 1966.

7. Metric entropy, widths, and superpositions of functions, Amer. Math. Monthly 69 (1962), 469-485.

8. L. Pontrjagin and L. Schnirelmann, Sur une propriété métrique de la dimension, Annals of Math. (2) 33 (1932), 156-162.

9. A. G. Vitushkin, Estimation of the complexity of tabulation problems, Moscow 1959. 10. Theory of the transmission and processing of information, Pergamon Press, New York, 1961 (English translation of 9).

Received October 24, 1966. This paper is related to part of the author's doctoral dissertation which was directed by Professor George G. Lorentz and supported by the United States Air Force through the Air Force Office of Scientific Research, under Contract No. AF 49(638)1401.

SYRACUSE UNIVERSITY AND

The General Electric Company 
